\title{
Natural Joints: Tribological performance on negatively charged surfaces
}

\author{
Zenon Pawlak ${ }^{*}$ and Aleksandra Mrela ${ }^{2}$ \\ ${ }^{1}$ Tribochemistry Consulting, Salt Lake City, UT 84117, USA, University of Economy, Biotribology Laboratory, Poland \\ ${ }^{2}$ Kujawy and Pomorze University in Bydgoszcz, Faculty of Technology, Poland
}

\begin{abstract}
This study examined the tribological performance of the normal articular cartilage, in relation to the surface of unhealthy one. Following sliding tests of a (cartilage/ cartilage) the worn surfaces wettability, and friction coefficient were studied. It is concluded that the phospholipids bilayers formation, a lubricant influenced the friction performance.
\end{abstract}

\section{Introduction}

The concept of "lamellar-electrostatic mechanism" is new [1] and compatible with the discovery of Brian Hills's hypothesis that lamellar phases of PLs and phospholipid bilayers are lubricants in natural joints [2]. The concept that boundary lubrication in joints was mediated by an active ingredient in synovial fluid (SF) was put forward by Linn and Radin [2] and Hills [3]. According to Hills and other authors, the surface amorphous layer (SAL) of cartilage contains surface-active phospholipids. Phosphatidylcholines, PC (over 40\%) sphingomyelin, SM ( 30\%) and phosphatidylethanolamines, PE ( 30\%) were subsequently identified in SAL and in the synovial fluid in content (lyso-phosphatidylcholines (LPC), phosphatidylethanolamines-based plasmalogens ( $\mathrm{PE}$ ), phosphatidylserines (PS), Phosphatidylglycerols (PG), and ceramides (Cer)) shown in Figure 1.

Surface active phospholipids play a vital role in joints tissue systems in large part due to an amphoteric nature that allows for varied structural properties. This amphoteric nature of phospholipids causes them to self-assemble into the classic arrangement that is the basis of all biological membranes. A Surface-Active Phospholipid Layer (SAPL) covers normal articular surfaces in a multi-bilayer structure. The bilayers serve to integrate interfacial functions between surfaces and have been a subject of much inquiry due to its tribological features. However, at sites of articular cartilage damage, the SAPL is absent because a suitable substrate upon which a SAPL can form does not exist [4-6].

This book is focused on the mechanism of natural lubrication by which the Phospholipids (PLs) in Articular Cartilage (AC) act as a lubricant. The physiological function of PLs is unique in preventing solid-solid contact and degradation of the articular surface. With AC, it is particularly important to have a model where PLs adsorbed on the surface of cartilage impart a number of highly desirable properties.

The importance of basic properties of the cartilage such as the surface energy, wettability, $\mathrm{pH}$, surface charge, and the amphoteric surface character was determined and turned out to be compatible with some features of joints lubrication.
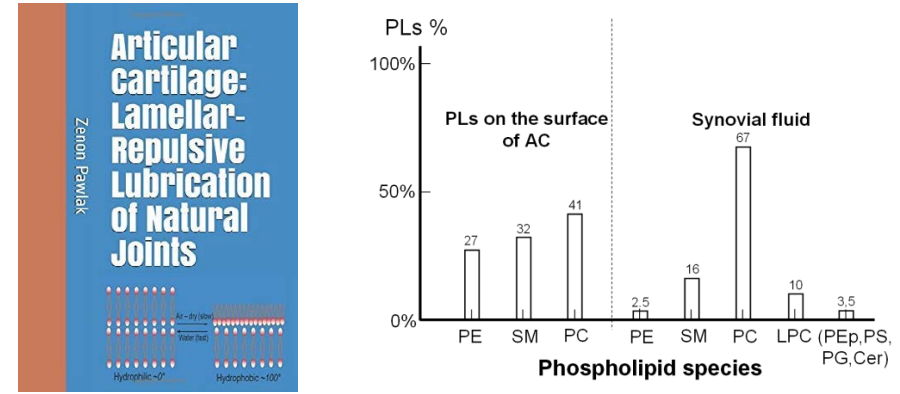

Figure 1. Book cover "Articular cartilage: Lamellar-repulsive lubrication of natural joints" and phospholipids contents on the surface of articular cartilage and in synovial fluid

\section{pH vs. friction of cartilage/cartilage surfaces}

In Figure 2, the friction coefficient measured for a partially depleted (curve 3 ) and completely depleted cartilage samples (curve 1) are compared with those of the healthy articular surface (curve 4). Curve (1) of completely depleted PLs from BC surface, wettability $37^{\circ}$, showing the absence of polycation $\left(-\mathrm{NH}_{3}^{+}\right)$and polyanion $\left(-\mathrm{PO}_{4}^{-}\right)$ groups. Curve (3) partially depleted PLs from BC surface, wettability $83^{\circ}$, showing much lower friction of polycation $\left(-\mathrm{NH}_{3}^{+}\right)$than polyanion $\left(-\mathrm{PO}_{4}^{-}\right)$charged surfaces. Curve (4) normal BC surface with wettability $103^{\circ}$; with a gradual increase in friction $\left(-\mathrm{NH}_{3}{ }^{+} \rightarrow-\mathrm{NH}_{2}\right)$ and a curve maximum at $\mathrm{pH} \sim 4.5$ (isoelectric point, IEP), after IEP leading to a negatively charged surface $\left(-\mathrm{PO}_{4} \mathrm{H} \rightarrow-\mathrm{PO}_{4}^{-}\right)$with a reduced friction coefficient. The friction coefficient of cartilage as a function of the $\mathrm{pH}$ (or charge density) is nonlinear for this amphoteric surface. Based on these experimental results it can be demonstrated that the cartilage friction is largely dependent on the electrostatic and electric polarization involving (as for water) interactions between two cartilage

${ }^{\star}$ Correspondence to: Zenon Pawlak, Tribochemistry Consulting, Salt Lake City, UT 84117, USA, University of Economy, Biotribology Laboratory, Garbary 2, 85-229 Bydgoszcz, Poland, E-mail: zpawlak@xmission.com

Received: May 25, 2018; Accepted: June 11, 2018; Published: June 15, 2018 


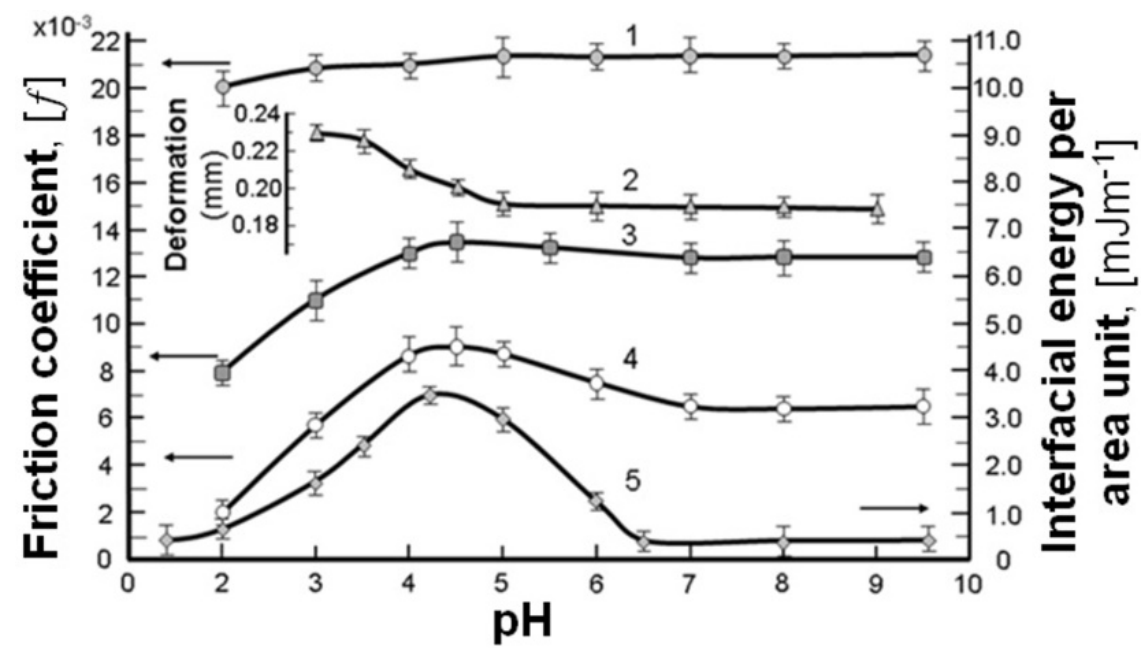

Figure 2. The $\mathrm{pH}$ effect on the friction of bovine cartilage, BC surface (curve 1,3,4), deformation of bovine cartilage surface (curve 2) [2] and interfacial energy energy of model membrane (used in the form of liposomes) formed by phosphatidylserine (curve 5). The standard deviation (SD) of the interfacial energy and the friction coefficient $f$ ranges from 10 to $15 \%$.

surfaces. Electrostatic repulsion between two cartilage surfaces, which favor the formation of a thicker water layer between the surfaces, shifting in this way the lubrication mode from boundary to a mixed or even hydrodynamic one, and distinctly reduces the friction between them.

\section{Deformation of the cartilage}

Curve (2) in Figure 2 displays deformation profiles of AC with the $\mathrm{pH}$, the deformation decreasing rapidly being positively charged before IEP $\sim \mathrm{pH} 5$ [2]. Deformation of the cartilage, upon compression, increased when the $\mathrm{pH}$ fell below $\sim 5$, whereas above this value it remained unchanged, curve (2). The deformation of the AC surface is discussed because it is related to several aspects of the lubrication of joints. The relation between deformability (or softening) and lower friction is observed at pH 2 to 4 , Figure 2, curve (4) and to a much lesser extent at $\mathrm{pH}$ 5-7.

\section{Interfacial energy}

Curve (5) shows the interfacial energy of the model membrane (used in the form of liposomes) formed by phosphatidylserine as a function of $\mathrm{pH}$ over the range 1.0 to 9.5 supporting the hypothesis of the amphoteric character of phospholipidic cartilage as determined by microelectrophoresis. Changes in interfacial energy interplay well enough with the amino- and phosphate-charged groups: thus, at a low $\mathrm{pH}\left(-\mathrm{NH}_{3}{ }^{+} \rightarrow-\mathrm{NH}_{2}\right.$, and after IEP ( $\left.\mathrm{pH} 4.5\right)$, whereas at a higher $\mathrm{pH}$ $\left(-\mathrm{PO}_{4} \mathrm{H} \rightarrow-\mathrm{PO}_{4}^{-}\right)$[7].

\section{Amphoteric nature of cartilage surfaces}

A relationship between the interfacial energy of the model membrane (curve 5) and the friction coefficients (curve 4) in a bovine cartilage pair vs. $\mathrm{pH}$ of the buffer solutions is demonstrated in figure 2. As the $\mathrm{pH}$ is varied, the interfacial energy of the liposomes' model membrane (curve 5) formed by phosphatidylserine and friction coefficient (curve 4) in a bovine cartilage pair reaches maximum on the curves, a remarkably similar amphoteric behavior occurring to that characterizing the isoelectric point, IEP.

Below the isoelectric point (max curve) of the cartilage surface and the liposomes' model phospholipid membrane, the surface is positively charged $(-\mathrm{NH} 3+)$ curve, with a gradual change of friction and interfacial energy as the $\mathrm{pH}$ shifts towards the IEP. After passing through the IEP, the surface charge gradually changes from positive (-NH3+) to negative (-PO4), while the surface friction changes from the attractive curve to a repulsive state curve. A similar trend was observed [8], using an amphoteric material, $\mathrm{SiO} 2$. Relatively small slops in the tails of curves 4 and 5 are a good reason to refer to these portions as the buffer regions with a very high buffer capacity $(\beta)$ imparted by the phospholipid, $\Delta \mathrm{H}+$ / $\Delta \mathrm{pH}=\beta$ [9]. This suggests that a portion of lubrication mechanism in joints can be described as a lamellar-repulsive hydration mechanism. We, therefore, conclude that lamellar slippage of bilayers and a shortrange repulsion between the interfaces of the negatively charged (- $\left.\mathrm{PO}_{4}^{-}\right)$ cartilage surfaces is a major facilitator of the low frictional properties of joint.

While the principal aim of the "lamellar-electrostatic mechanism" is to provide an understanding of the meaning of the surface-active state (lamellar bodies), the other is to highlight the solid PL bilayers that possess low surface energy at $\mathrm{pH} \sim 7.4$, when applied to the body. The understanding of $\mathrm{AC}$ amphoteric surface will be essential for the effective repair and regeneration of the degraded knee joint. It has been confirmed experimentally that phospholipidic multi-bilayers are essentially described as lamellar frictionless lubricants protecting the surface of the joints against wear [9].

Friction between Phospholipid (PL) bilayers attached to cartilage surfaces is then considered including discussion on the recent observation of an extreme friction reduction as a consequence of a less charged hydrophilic cartilage surface. It is proposed that the highly efficient lubrication occurring in natural joints arises from the presence of negatively charged cartilage surfaces. The lamellarrepulsive mechanisms for the reduction of friction is supported by phospholipid lamellar phases and charged macromolecules residing between contacting cartilage surfaces $[10,11]$.

In conclusion, it can be stated that the lamellar-electrostatic lubrication mechanism, a new approach to understanding natural lubrication, is challenging and deserves to be studied.

\section{References}

1. Pawlak Z (2018) Articular Cartilage: Lamellar-Repulsive Lubrication of Natural Joints Kindle Direct Publishing. PP. 171.

2. Linn FC, Radin EL (1968) Lubrication of animal joints. 3. The effect of certain chemica alterations of the cartilage and lubricant. Arthritis Rheum 11: 674-682. [Crossref] 
3. Hills BA, Butler BD (1984) Surfactants identified in synovial fluid and their ability to act as boundary lubricants. Ann Rheum Dis 43: 641-648. [Crossref]

4. Hills BA (1989) Oligolamellar lubrication of joints by surface active phospholipid. $J$ Rheumatol 16: 82-91. [Crossref]

5. Sarma AV, Powell GL, LaBerg M (2001) Phospholipid composition of articular cartilage boundary lubricant. J Orthop Res 19: 671-676. [Crossref]

6. Kosinska MK, Liebisch G, Lochnit G, Wilhelm J, Klein H, et al. (2013) A lipidomic study of phospholipid classes and species in human synovial fluid. Arthritis Rheum 65: 2323-2333. [Crossref]

7. Pawlak Z, Figaszewski ZA, Gadomski A, Urbaniak W, Oloyede A (2010) The ultra-low friction of the articular surface is $\mathrm{pH}$-dependent and is built on a hydrophobic underlay including a hypothesis on joint lubrication mechanism'. Tribology International 43: 1719-1725.

8. Pawlak Z, Yusuf KQ, Pai R, Urbaniak W (2017) Repulsive surfaces and lamellar lubrication of synovial joints. Arch Biochem Biophys 623-624: 42-48. [Crossref]

9. Marti A, Hahner G, Spencer ND (1995) Sensitivity of frictional forces to $\mathrm{pH}$ on ananometer scale: A lateral force microscopy study. Langmuir 11: 4632-4635.

10. Pawlak Z, Urbaniak W, Hagner-Derengowska M, et al. (2015) The Probable Explanation for the Low Friction of Natural Joints. Cell Biochem Biophys 71: 1615-1621. [Crossref]

11. Pawlak Z, Gadomski A, Sojka M, Urbaniak W, Beldowski P (2016) The amphoteric effect on friction between the bovine cartilage/cartilage surfaces under slightly sheared hydration lubrication mode. Colloids Surf B Biointerfaces 146: 452-58. [Crossref]

Copyright: ( 2018 Pawlak Z. This is an open-access article distributed under the terms of the Creative Commons Attribution License, which permits unrestricted use, distribution, and reproduction in any medium, provided the original author and source are credited. 